\title{
Facilitating Message Exchange though Middle Agents
}

\author{
Terry R. Payne, Massimo Paolucci, Rahul Singh \& Katia Sycara \\ Carnegie Mellon University \\ The Robotics Institute \\ 5000 Forbes Avenue \\ Pittsburgh PA 15213, USA \\ \{terryp, paolucci, kingtiny, katia\}@cs.cmu.edu
}

\begin{abstract}
To utilize services provided by other agents, a requesting agent needs to locate and communicate with these service providers. Specifically, in order to interoperate with the providers, the requesting agent should know: 1) the service provider's interface; 2) the ontology that defines concepts used by the provider; and 3) the agent communication language (ACL) the agent uses so that it can parse and understand the communication. Currently deployed Multi-Agent Systems (MAS) encode the interface description and the ontology within a service provider's capability description (or advertisement) that is registered with a Middle Agent; however, this assumes a common ACL between communicating agents. We demonstrate how agents can communicate with each other using a template-based shallow parsing approach to constructing and decomposing messages, thus relaxing assumptions on the ACLs and message formats used.
\end{abstract}

\section{Categories and Subject Descriptors}

I.2.11 [Artificial Intelligence]: Distributed Artificial Intelligence-Multiagent systems; I.2.11 [Artificial Intelligence]: Distributed Artificial Intelligence-Coherence and coordination

\section{General Terms}

Design

\section{INTRODUCTION}

Multi-Agent Systems (MAS) that contain more than a trivial number of heterogeneous agents typically rely on infrastructures that support service discovery and agent interoperation. A Middle Agent [4, 7] provides lookup services that facilitate the discovery of agents with specific capability descriptions; it may also mediate communication between agents. Problems arise when an agent requesting a service (i.e. a service requester) has no prior knowledge of the format of the message expected by the service provider, or of

Permission to make digital or hard copies of all or part of this work for personal or classroom use is granted without fee provided that copies are not made or distributed for profit or commercial advantage and that copies bear this notice and the full citation on the first page. To copy otherwise, to republish, to post on servers or to redistribute to lists, requires prior specific permission and/or a fee.

AAMAS'02, July 15-19, 2002, Bologna, Italy.

Copyright 2002 ACM 1-58113-480-0/02/0007 ...\$5.00. how to interpret the response. In such cases, although service requesters may discover providers via a Middle Agent, they may not be able to communicate with them. Agent Communication Languages (ACLs) such as FIPA [2] propose the adoption of a common ACL to which all agents adhere. Whilst such languages specify the type of communicative action that the agents perform, as well as the sender and other transport information, they do not always provide a specification of the content of the message. We present a shallowparsing template approach which relaxes the constraint that agents share a common language for describing the content and format of messages. Message templates can be used in combination with advertised capability descriptions to construct and exchange messages between agents. Thus, the only assumptions made are: (1) that agents can interact with the Middle Agent (i.e. a common Middle Agent communications protocol is used, and agents adhere to a common capability description language (CDL) $[7,1])$; and (2) that they share the same ontology as the provider. Crucially though, the two agents do not have to share the same ACL.

\section{MEDIATION AND MIDDLE AGENTS}

Middle Agents [6] assist in the discovery of service providers based upon a desired service capability description. The behavior of a Middle Agent is determined by its matching process and its interaction protocols: the matching process is dependent on the capability description language (CDL) used; whereas the interaction protocol determines how agents interact with a Middle Agent, and (in some cases) how the Middle Agent mediates agent communication. Typically, when agents appear within a MAS, they advertise their capabilities with a Middle Agent. However, the method used for discovering and interacting with a service provider can vary depending on the type of Middle Agent used. For example, Brokers or Facilitators [4] mediate between requesters and providers by querying services whose advertisements match a requester's service query. Resulting messages are then sent from the provider to the requester via the $\mathrm{Fa}$ cilitator. This contrasts with Matchmakers $[6,7,3]$ ), which do not participate in the agent-to-agent communication process; but rather match service requests with advertisements, and return these matches to the requesters.

The location of services is one of several challenges encountered by agents within a MAS. Once a provider has been located and selected, it should be queried and its answer should be interpreted. The advertisement of the provider can be used to assist in the construction of the query message and interpretation of the reply message. The shallow- 
parsing template approach, adopted here, is based on the inclusion of preformatted message templates within the advertised capability description that can be used to map advertisements into queries, and to extract information from replies. These templates define the format of the messages as character sequences, and denote the location of parameter values within the message using placeholders. Provider's Advertisement

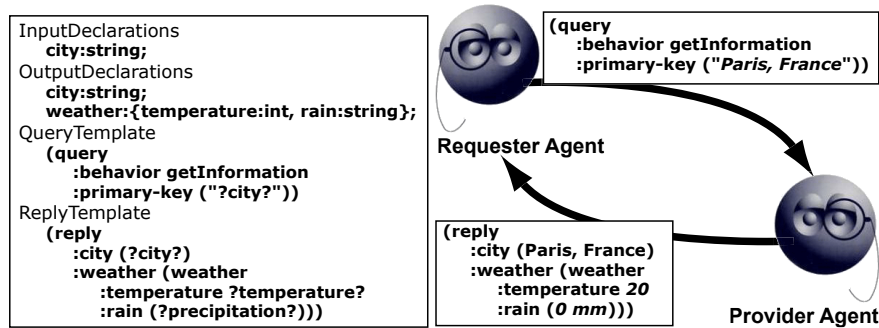

Figure 1: Generating messages from templates.

Examples of query and reply templates are given in Fig. 1 , where the fields query and reply contain templates for query and reply messages respectively. The query template represents a sample query with unspecified input parameters, delimited with '?' characters, that specifies where input parameters should appear within outgoing messages. The reply template can be used to identify where output parameters appear within incoming messages, so that these messages can be parsed and decomposed. The advertisement in Fig. 1 describes how an agent may be queried to provide weather information about a given city. It is not necessary for the requesting agent to understand the content of the query it submits, or the content of the reply it receives. It simply replaces the placeholder ?city? with the name of the desired city, i.e. Paris, France. By combining information about each parameter (i.e. data type, range of possible values or links to semantic concepts) with the reply template, the requester can decompose the reply from the service provider into value-attribute tuples. This is achieved by comparing the template with the reply, and attributing the unmatched string sequences to the corresponding parameter placeholders. For example, the reply in Fig. 1 can be decomposed to generate three tuples: [city, (Paris, France)] [temperature, (20)] and [rain, (0 mm)].

This approach naturally lends itself to peer-to-peer communication within a Matchmaker-based MAS. It can also be applied to a Facilitated MAS provided that the following four steps are satisfied:

(1) The service requester submits a query to the Facilitator. This query contains parameters representing a subset of the requesters knowledge, the desired outputs, and a query template that is used to decompose the query into tuples.

(2) The Facilitator locates a service provider that offers the desired service. The tuples are used to construct a service request, which is then matched against advertised services to select a service provider.

(3) The Facilitator constructs and submits a query to the service provider. The query is constructed using the tuples and the provider's query template.

(4) The service provider responds by sending a reply to the Facilitator, which constructs a reply for the service requester. The Facilitator uses the provider's reply template to decompose the reply into tuples, when are then used to construct a reply to the requester based on the requester's reply template.

At this stage the transaction between the requester and the provider is complete and the agent has the information that it was seeking. It can now proceed to solve the problem that originally prompted the transaction.

\section{DISCUSSION}

Although the shallow-parsing template approach relaxes the assumption that two communicating agents must share a common agent communication language, it does impose certain restrictions on the type of messages that can be exchanged. For example, the use of message templates establishes an upper bound on the expressivity of agent communication, by restricting the number of messages that two agents can exchange to those specified by the templates in the advertisement. In order to support a richer level of communication, agents should agree upon an ACL based upon social semantics [5]. This allows agents to express messages in which unrestricted conversations can take place. However, in most cases, it is extremely difficult to construct agents that exploit this, and currently there are no agents that interoperate with each other through unconstrained communication.

The use of message templates imposes its own assumptions, namely that agents can parse and interpret the templates, and that the templates will be included within the capability descriptions. However, it is currently assumed that agents within the MAS share the same CDL, and hence can utilize a Middle Agent's discovery services. Thus, the CDL can be augmented to include optional message templates to support agent communication when there is a mismatch with assumed ACLs. The shallow-parsing template approach also assumes that all agents share a common ontology so that they understand without ambiguities the meaning of terms in the request and reply.

The adoption of the templates lowers the requirement for agent interoperation, allowing agents in an open MAS to communicate despite the absence of previous agreements on ACL and message format.

\section{ACKNOWLEDGMENTS}

The research was funded by the Defense Advanced Research Projects Agency as part of the DAML program under AFRL contract F30601-00-2-0592 to Carnegie Mellon University. Special thanks goes to Martin van Velsen for his contribution towards some of the ideas presented here.

\section{REFERENCES}

[1] A. Ankolekar, M. Burstein, J. Hobbs, O. Lassila, D. Martin, S. McIlraith, S. Narayanan, M. Paolucci, T. Payne, K. Sycara, and H. Zeng. Daml-s: Semantic Markup for Web Services. In International Semantic Web Working Symposium, 2001.

[2] FIPA. Foundation for Intelligent Physical Agents. http://www.fipa.org/, 1997.

[3] M. Genesereth and S. Katchpel. Software Agents. Communications of the ACM, 37(7):48-53, 1994.

[4] D. Martin, A. Cheyer, and D. Moran. The Open Agent Architecture; A Framework for building Distributed Software Systems. Applied Artificial Intelligence, 13(1-2):92-128, 1999

[5] M. P. Singh. A Social Semantics for Agent Communication Languages. Technical Report TR-99-03, 22, 1999.

[6] K. Sycara, K. Decker, and M. Williamson. Middle-Agents for the Internet. In Proceedings of IJCAI-97, January 1997.

[7] K. Sycara, M. Klusch, S. Widoff, and J. Lu. Dynamic service matchmaking among agents in open information environments. SIGMOD Record (ACM Special Interests Group on Management of Data), 28(1):47-53, 1999. 